\title{
Atmospheric Impairments and Mitigation Techniques for High-Frequency Earth-Space Communication System in Heavy Rain Region: A Brief Review
}

\author{
Shi Jie Seah', Siat Ling Jong ${ }^{2, *}$, Hong Yin Lam³ \\ ${ }^{1}$ Department of Communication Engineering, \\ Faculty of Electrical and Electronic Engineering, Universiti Tun Hussein Onn Malaysia, Batu Pahat, 86400, Johor, \\ MALAYSIA \\ ${ }^{2}$ Wireless and Radio Science Centre \\ Faculty of Electrical and Electronic Engineering, Universiti Tun Hussein Onn Malaysia, Batu Pahat, 86400, Johor, \\ MALAYSIA
}

${ }^{3}$ Faculty of Engineering Technology, Universiti Tun Hussein Onn Malaysia, 84600 Hub Pendidikan Tinggi Pagoh, KM1, Jalan Panchor, Johor, MALAYSIA

\section{*Corresponding Author}

DOI: https://doi.org/10.30880/ijie.2019.11.03.017

Received 24 July 2019; Accepted 31 July 2019; Available online 3 September 2019

\begin{abstract}
This work surveys the atmospheric impairments that affect a satellite link operating in a high-frequency band, such as $\mathrm{Ka}$ and $\mathrm{Q} / \mathrm{V}$ bands, particularly in heavy rain regions. The impacts of hydrometeors and cloud attenuation are emphasised and discussed along with the contribution of gases and scintillation to signal fade. Also, propagation impairment mitigation techniques are reviewed from the perspective of satellite operators in heavy rain areas.
\end{abstract}

Keywords: Cloud attenuation, Prediction model, Rain attenuation, Scintillation, Fade mitigation technique.

\section{Introduction}

Atmospheric effects such as gaseous absorption, rain attenuation, cloud attenuation and tropospheric scintillation are considered major impairments to terrestrial and earth-to-satellite signal transmission at frequencies above $10 \mathrm{GHz}$. The current demand for high-speed communication with larger bandwidths is the main reas on for the interest in using the $\mathrm{Ka}$ and $\mathrm{Q} / \mathrm{V}$ frequency bands. However, these frequency bands could severely dimin ish the performance of systems [1]-[3]. In this respect, the carrier-to-noise ratio $(\mathrm{C} / \mathrm{N})$ and system availability, two aspects of primary system performance, are key parameters affecting the quality of service (QoS). Atmospheric gases, rain, cloud, scintillation and depolarisation are factors that weaken the carrier power, while other factors, such as atmospheric gases, rain, terrestrial interference and surface emissions, increase the noise power of the system [4]. Atmospheric effects, especially rain attenuation, are expected to be more severe in tropical and equatorial climatic regions [5], [6] as these areas experience wet weather throughout the year. In addition, precipitation may cause occurrences of scintillation when a signal degrades during the rain events. This paper discusses and reviews previous work on propagation effects at future high-frequency bands such as Ka and Q/V. The paper is structured as follows. First, the effects of scintillation are reviewed in Section 2, and then attenuation due to rain is discussed extensively in Section 3. Section 4 presents a 
brief review of cloud attenuation, and Section 5 reviews water vapor attenuation. Finally, Section 6 summarises important propagation impairment mitigation techniques, and Section 7 draws some conclusions.

\section{Scintillation and Attenuation}

Scintillation refers to changes in the refractive index due to shifts in the amplitude, phase or angle of arrival of a clear atmosphere [7]. There are differences between scintillation and rain attenuation; scintillation leads to rapid fluctuation as soon as a turbulent atmosphere degrades the signal, while rain attenuation leads to slower fluctuation in signal strength. Scintillation is triggered by rapid temporal changes in the properties of the medium and geometry of propagation as well as variation in the spatial properties of the propagation medium or geometry [8]-[10]. Hence, it is necessary to differentiate between the effects of rain attenuation and scintillation. These effects can be separated by employing the filtering method discussed in [7]. A Butterworth low-pass filter and a high-pass filter were used normally to measure rain attenuation and scintillation, respectively. The power spectrum of the time series was analysed before the filtering process to estimate the range of frequencies in which the physical phenomenon increased or decreased amp litude. Fig. 1 shows the average power spectral density of 33 events observed in Milan [7], which can be used to study the relationship between scintillation and rain attenuation. It is clearly shown from the Fig. 1 that the overlapping effects of scintillation and rain attenuation occurred after which the average theoretical $-20 \mathrm{~dB} / \mathrm{decade}$ slope effectively increases until the cut-off frequency of $0.02-0.03 \mathrm{~Hz}$.

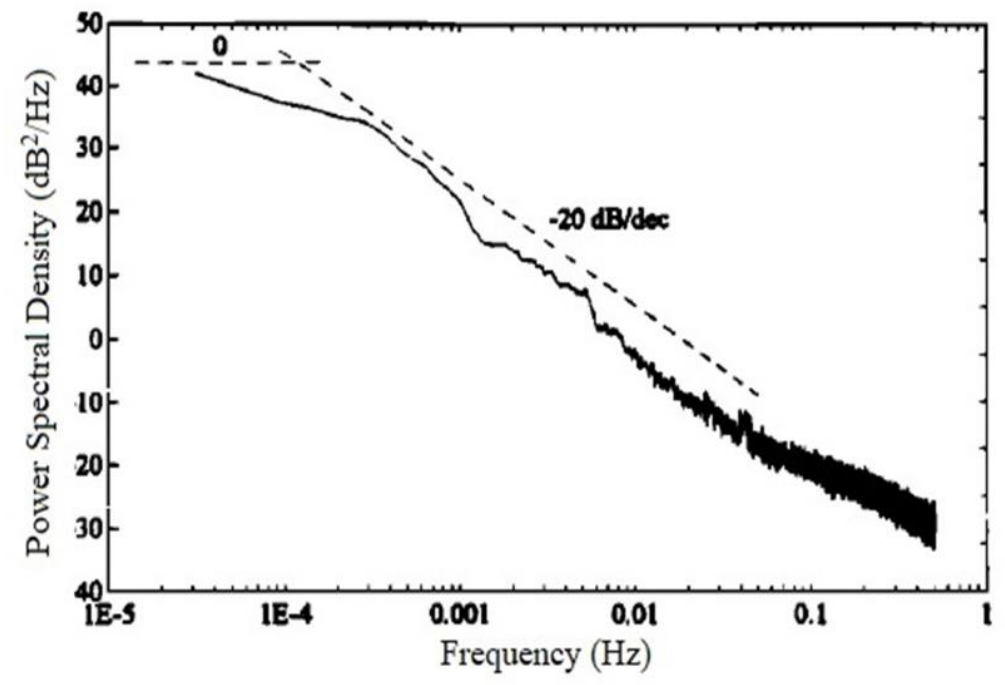

Fig. 1. Power spectral density of 33 events in Spino d'Adda [7].

The phenomena of rain attenuation and scintillation can be distinguished if the cut-off frequency is identified. To do so, a low-pass filter was used to remove fast fluctuations from the raw time series of the strength of received signals. Details regarding this process are provided in [7]. Furthermore, high-power rain attenuation and isolated scintillation are removed by a high-pass filter to obtain a high-pass scintillation time series. Fig. 2 shows the scintillation time series after the filtering process, revealing that the scintillation has lower power and higher frequencies compared to rain attenuation.

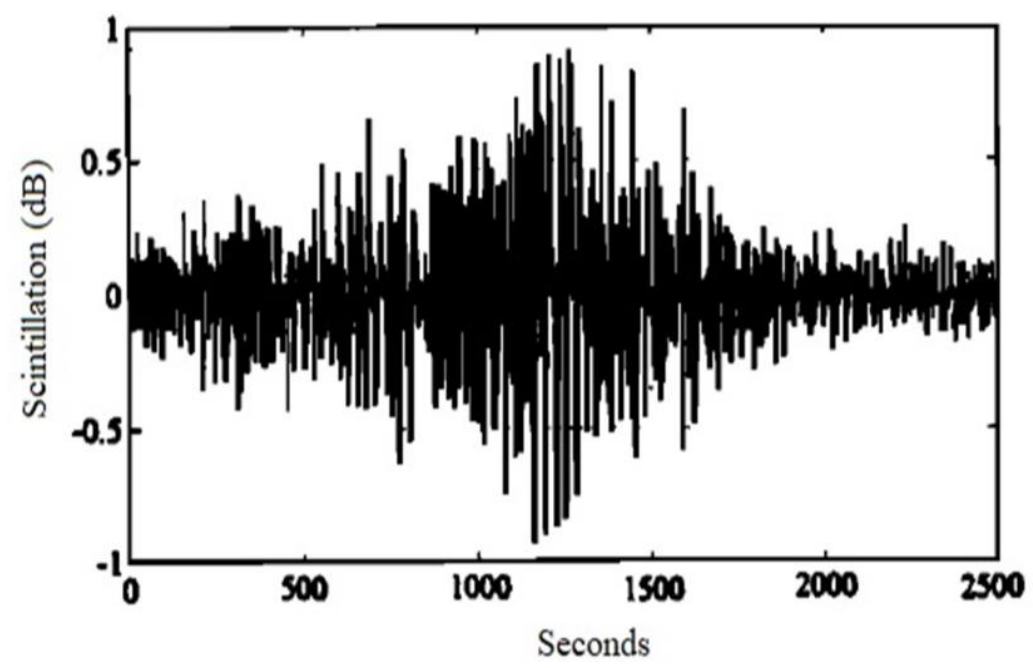

Fig 2. Example of time series scintillation in Spino d'Adda [7]. 
Tropospheric turbulence that causes rapid fluctuation in an earth-space path is normally managed with the structure constant, a parameter that allows one to determine the severity of rapid fluctuations in signal propagation. Researchers have proposed a technique for obtaining the refractive index structure constant of attenuation and the structure constant relation [9]. Fig. 3 shows a scatter plot of structure constant against attenuation of a rain event on August 13, 2008. The turbulent layer model is usually used to study the correlation between scintillation and rain attenuation. However, a random model was proposed in [10] to explain the effects of amplitude scintillation on rainfall. Such results show that amplitude scintillation becomes more critical when rain fading is high. Other researchers have studied the relationship between scintillation and rain attenuation in the context of $\mathrm{Ku}$-band satellite signals, finding that a thin-layer turbulence model is valid for studying signal scintillation [8], [9]. The turbulences effects related with rain events may be unlike for different climatic regions. This is evidenced from the work obtained from [9] for Ku-band in tropical region, in which it has stronger turbulences due to higher standard deviations of scintillation fluctuations as compared to Ku-band link in temperate region reported by Matricciani et.al [11].

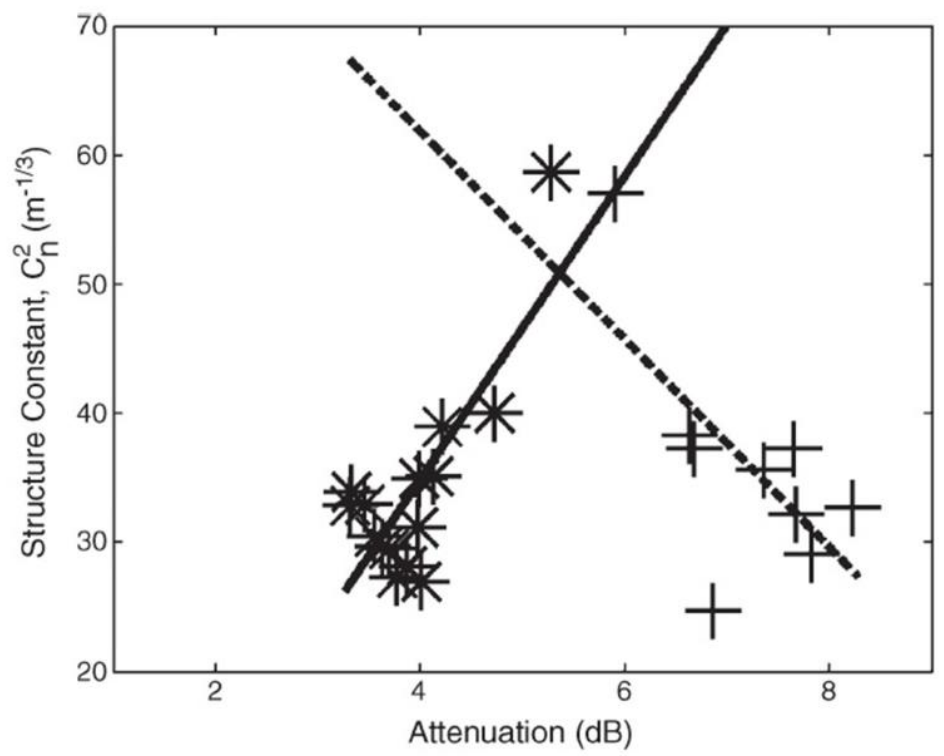

Fig 3. Scatter plot of structure constant against rain attenuation in India [9].

\section{Rain Attenuation}

Among the various types of atmospheric attenuation, rain attenuation is the major phenomenon resulting in propagation impairment of earth-space communication links. The absorption and scattering of electromagnetic waves produce significant fading or signal attenuation when radio waves propagate through rainy regions. Although rain attenuation has been the subject of investigation for several decades, its impact is becoming increasingly significant as communication systems move towards higher frequencies and wavelengths are comparable to the size of raindrops, which leads to a scattering effect. This section reviews several factors that affect rain attenuation as well as importan $t$ developments in the field.

\subsection{Factor Affecting Rain Attenuation}

For any path, rain attenuation depends on the propagation parameters, which include rainfall rate, frequency, elevation angle, path length and raindrop size distribution (DSD). Thus, these factors are discussed in the following subsection.

\subsubsection{Rainfall Rate and Specific Attenuation}

Rainfall rate is the most basic parameter used to determine the attenuation induced by rain along the terrestrial and earth-space paths. The power law relation between specific attenuation, $\gamma$, and rain rate, $R$, is generally used to calculate specific attenuation or attenuation per unit distance [12], and it is included in the International Telecommunication Union-Radio communication (ITU-R) P.838-3 recommendation [13], as shown in equation (1).

$$
\gamma=k R^{\alpha}
$$

where the coefficient of $k$ and $\alpha$ is determined as a function of frequency, polarisation, elevation angle and scattering calculations derived from the DSD and raindrop shape. The values of $k$ and $\alpha$ with respect to horizontal and vertical polarisation are shown in Table 1 of the ITU-R 838-3 recommendation [13]. These values are a function of frequency, polarisation and DSD, and they can range from 1-1000 GHz based on equations (2), (3), (4) and (5). 


$$
\begin{gathered}
\log _{10} k=\sum_{j=1}^{4}\left(a_{j} \exp \left[-\left(\frac{\log _{10} f-b_{j}}{c_{j}}\right)^{2}\right]\right)+m_{k} \log _{10} f+c_{k} \\
\propto=\sum_{j=1}^{5}\left(a_{j} \exp \left[-\left(\frac{\log _{10} f-b_{j}}{c_{j}}\right)^{2}\right]\right)+m_{\propto} \log _{10} f+c_{\propto} \\
k=\left[k_{H}+k_{V}+\left(k_{H}-k_{V}\right) \cos ^{2} \theta \cos 2 \tau\right] / 2 \\
\alpha=\left[k_{H} \alpha_{H}+k_{V} \alpha_{V}+\left(k_{H} \alpha_{H}-k_{V} \alpha_{V}\right) \cos ^{2} \theta \cos 2 \tau\right] / 2 k,
\end{gathered}
$$

where $f$ refers to frequency (GHz), $k$ refers to either the constant $k_{H}$ or $k_{V}$ and $\alpha$ refers to either the constant $\alpha_{H}$ or $\alpha_{V}$. The values for constants $k$ and $\alpha$ with respect to horizontal and vertical polarisations are shown in Tables 1-4 in [13].

A number of frequencies with different values have been chosen to evaluate the performance of the prediction model described in [14] and illustrated in Fig. 4. It is clear that higher frequencies may lead to higher attenuation and that rain attenuation decreases with the increases of elevation angle. The increase of frequency leads to higher attenuation may be due to the raindrop size. It is reported that the raindrop size around $1.67 \mathrm{~mm}$ on the average diameter would cause significant attenuation [15].

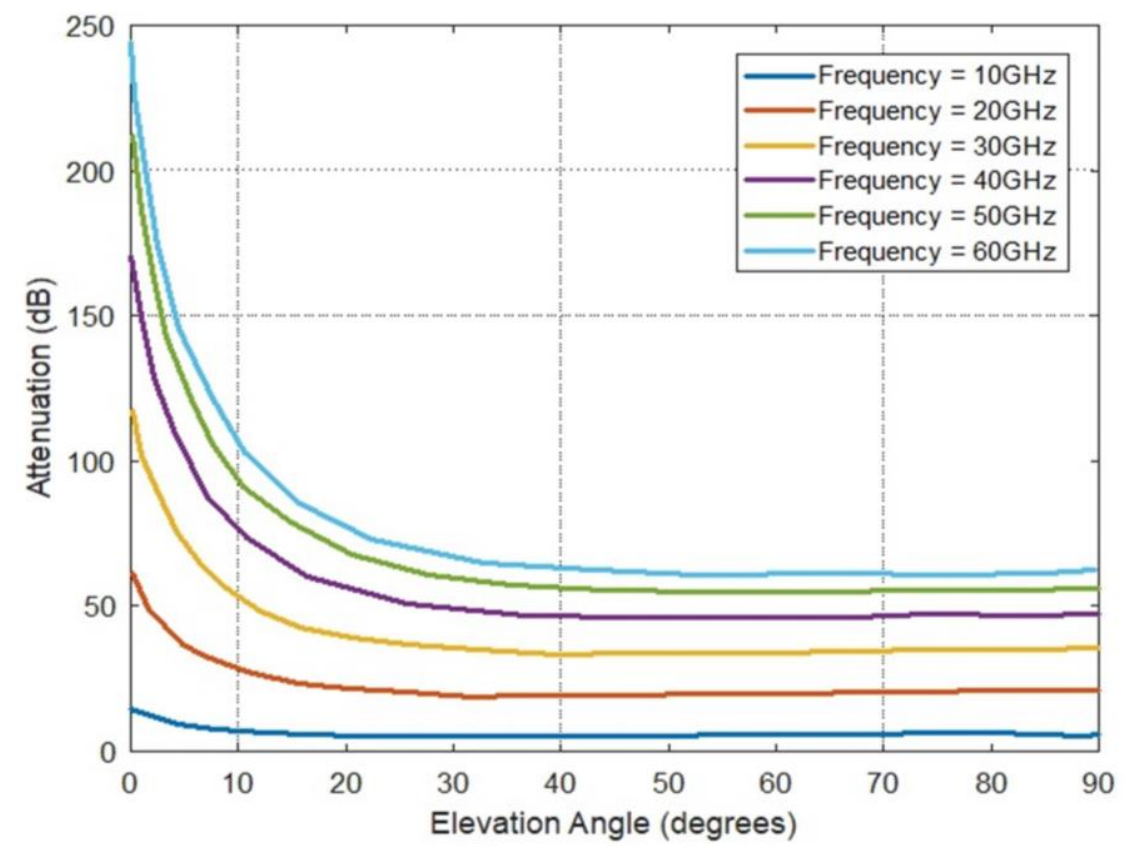

Fig. 4. The impact of attenuation as a function of frequency and elevation angle [14]

\subsubsection{Raindrop Size Distribution}

DSD plays the most crucial role in estimation of the attenuation of radio waves. As it can vary between regions depending on the peculiarities of local climates, it is best to estimate specific attenuation based on fundamental measurements of DSD. Unfortunately, DSD measurements are rarely available, so a DSD model is usually employed instead. Common, well-established DSD models include log-normal, exponential and gamma models. Various parameters determine the accuracy of DSD models, such as the intercept parameter $\left(N_{0}\right)$, shape parameter $(\mu)$ and slope parameter ( 1 ). Based on these, many studies have attempted to develop the best DSD model for various contexts, including heavy rain regions. For example, one study analysed truncated two-parameter gamma models based on measured DSD in Singapore [16], and others examined the statistical characteristics and variability of DSD with respect to rain attenuation in Kuala Lumpur, Malaysia [17], [18].

\subsubsection{Path Length}

The effect of rain on the length of the communication path is another important factor in determining link attenuation due to precipitation. This factor is usually referred to as the effective path length, $L_{e f f}$. The length is affected by the spatial and horizontal structure of rain cells or a rain field, and it is generally determined based on the following equation:

$$
A=\gamma \cdot L_{e f f}
$$


where $A$ refers to rain attenuation and $\gamma$ indicates the specific rain attenuation. From equation (6), it can be concluded that the rain attenuation increases with the increasing of effective path length.

\subsubsection{Elevation Angle}

Path length is interrelated with elevation angle; as the elevation angle increases, the path length of the radio link affected by rain decreased as a function of the look angle. Hence, the availability of communication will be improved. If the elevation angle is low, it is expected that a longer path link will be affected by rain, hence reducing the QoS of the communication system. Also, a lower elevation angle leads to scintillation effects. Fig. 5 plots the impact of elevation angle based on the ITU-R P.618-13 model [19].

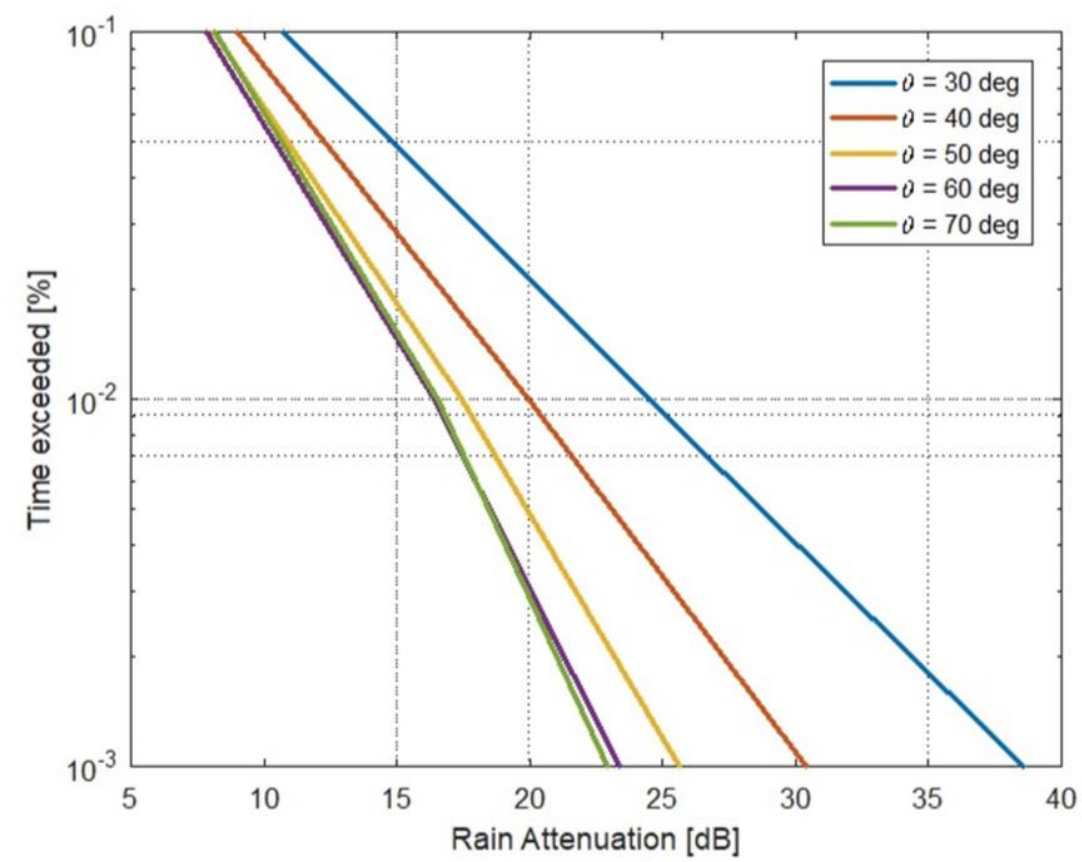

Fig. 5: Cumulative distribution of rain attenuation based on the ITU-R model [19]

\subsection{Rain Attenuation Prediction Model}

Various rain attenuation prediction models have been developed in the literature [20]. Most models were developed from physical and empirical models based on temperate regions, but some were developed based on measurements from heavy rain regions [21], [22]. To date, the ITU-R P.618-13 recommendation has been the most widely used model within the propagation community. However, another rain field model, MultiEXCELL [23] is considered as one of the most complete models for simulating and generating the temporal and spatial characteristics of precipitation for propagation applications. It is aims to improve the simple expressions of earlier model, named EXCELL model [24]. Prior to the development of the MultiEXCELL model, the SC EXCELL model was used to separately and tackle the impacts of stratiform and convective rain [25]. For heavy rain regions, a different rain attenuation prediction model based on the path adjustment factor was also proposed [26].

The synthetic storm technique (SST) model, developed by Matricciani [27] to obtain rain attenuation time series through conversion of recorded time series of rain rate, is also considered as a reliable model. As shown in Fig. 6, in the SST model, the structure of rain is modelled in to two layers: a rain layer (layer A) and a melting layer (layer B) [27]. In layer $\mathrm{A}$, the vertical rain rate, $R$, is uniform and measured using a rain gauge. However, it is much more complex to characterise layer B, as various assumptions must be made. Specifically, it must be assumed that the hydrometeor mass is constant during melting, there is a one-to-one correspondence between the rain and melting layers, the flux of hydrometeors is the same for both layers and the specific attenuation of layer B can be calculated if it is produced by raindrops with the same volume of melting hydrometeors. The physical and mathe matical details of the SST model are presented in [27]. A subsequent study reported that SST performs better than the ITU-R model when estimating fade duration statistics but performed similarly when predicting fade slope statistics [28]. However, [29] reported that a 1min rain rate time series cannot be used to predict fade durations shorter than 64-128 s. 


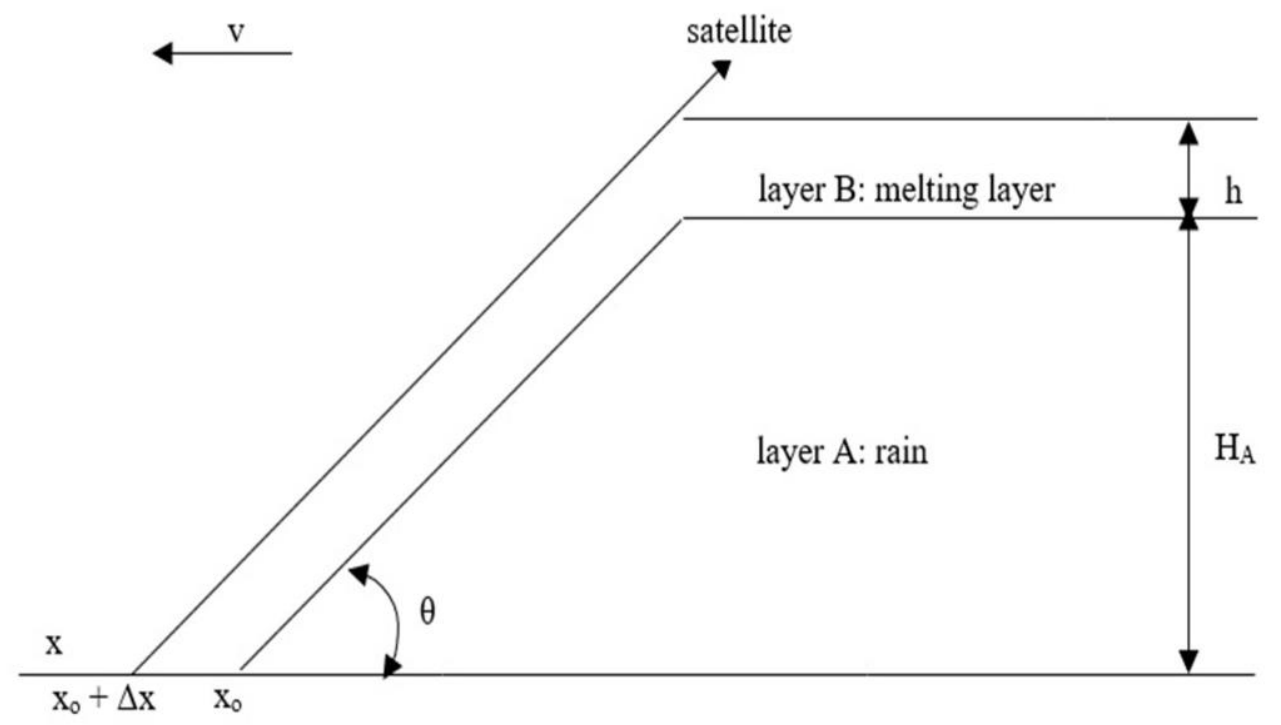

Fig. 6. Vertical structure of precipitation [27]

\subsection{Rain Attenuation Characteristics in a Heavy Rain Region}

As mentioned above, the unique precipitation characteristics of equatorial and tropical regions play an important role in determining the performance of satellite communication systems in these areas. Various studies by propagation scholars have focused on rain attenuation over the past few decades. However, there is still a lack of properly set up measurement campaigns or experimental work at high-frequency bands, such as the Ka band and above. The only exception is described in [30] in 2015, the European Space Agency launched a measurement campaign concerning a Ka-band satellite link located in the southern area of the Peninsular Malaysia.

It is important to consider the local climatic characteristics regarding rain in heavy rain regions to ensure proper design of satellite systems for the promised availability. For example, in equatorial Malaysia, the rainfall distribution is influenced by northeast monsoons and southwest monsoons, which occur from December to March and from June to September, respectively. Moreover, there are two short inter-monsoon seasons between these two seasons; the presouthwest season occurs from April to May, and the pre-northeast season occurs from October to November [31]The most intense precipitation experienced by radio links occurs during these inter-monsoon periods [32], [33].

In addition to seasonal effects, diurnal variation in rainfall is a critical feature of the geophysical and atmospheric dynamics that control rain fall. It is particularly significant in heavy rain regions like Malaysia, which are characterised by significant diurnal variations throughout the day [34]. The time period of a day has been divided into four-time intervals; 00:00-06:00, 06:00-12:00, 12:00-18:00 and 18:00-24:00 for diurnal variation of rain attenuation analysis.

Stratiform and convective types of rainfall have different spatial structures, space-time evolutions and impacts on radio wave propagation [25], [34]. Thus, type of rain (i.e. stratiform or convective rain) falls in a given area has a distinct impact on rain attenuation predictions. Stratiform rainfall develops at the cloud layer in low -pressure areas containing ice. In the tropospheric layer, ice crystals are formed by clouds. Snowflakes develop when the ice crystals become large enough to slowly fall and jo in with other such crystals until they reach the melt ing layer. Snow falls more rapidly as its size increases. Raindrops are formed when snow melts as it falls through air with a temperature of over $0^{\circ} \mathrm{C}$. Conversely, convective rain is generated when warm, moist air comes into contact with colder air at a high altitude. The air condenses into clouds as soon as the air is cooled below its dew point.

\section{Cloud Attenuation Models}

As the operating frequency of satellite communication increases, cloud attenuation begins to contribute to signal degradation. Clouds consist of water droplets, ice and oxygen molecules. The type and size of a cloud determine the cloud's capacity for water droplets, either in liquid or solid (i.e. ice) form. The different types of clouds - cumulus, cumulonimbus, stratus, stratocumulus, nimbostratus, altostratus, altocumulus and cirrus - are distinguished by their height. The properties and probability of occurrence of each type of cloud (except cirrus clouds, which have too many ice crystals [35]) allow researchers to characterise cloud attenuation. The cloud types can also be characterized by the concentration, liquid water content and droplet diameter which has been tabulated in Table 1. 
Table 1 - Characteristics of cloud types [1]

\begin{tabular}{cccc}
\hline Cloud Type & Concentration & Liquid water & Average radius \\
\hline Fair-weather cumulus & 300 & 0.15 & 4.9 \\
Stratocumulus & 350 & 0.16 & 4.8 \\
Stratus (over land) & 464 & 0.27 & 5.2 \\
Altostratus & 450 & 0.46 & 6.2 \\
Stratus (over water) & 260 & 0.49 & 7.6 \\
Cumulus congestus & $2-7$ & 0.67 & 9.2 \\
Cumulonimbus & 72 & 0.98 & 14.8 \\
Nimbostratus & 330 & 0.99 & 9.0 \\
\hline
\end{tabular}

Several cloud attenuation models, including ITU-R P.840-7 [36], have been developed to calculate cloud attenuation. The Salonen Uppala model [37], which was developed in 1991, is the best-known cloud attenuation model. However, the scope of application of previous cloud models is inadequate, and because they assumed uniform horizontal distribution, they are limited in their ability to estimate the impact of clouds on attenuation. Therefore, a new model for predicting cloud attenuation, the Stochastic Model of Clouds, was developed by [38]. Th is model synthesis ed three-dimensional cloud fields in high resolution. In addition, researchers performed a statistical study of cloud attenuation of Ka-band satellite signals [39]. In this study, cloud events were distinguished from rain events using the differentiating technique described in [40]. Another stochastic dynamic model was developed in 2017 [41]. This model generates integrated liquid water content (ILWC) fields by merging the space- and time-related behaviour of ILWC. Recently, a more precise method of identify ing cloud attenuation in tropical regions was proposed [42] based on data obtained from a heavy rain region and the relationship between ILWC and precipitable water vapourwas investigated.

\section{Water Vapor}

Water vapor is another factor that induces attenuation of earth-satellite links. Relative humidity is mandatory to determine water vapor density and later to be used in combination with pressure and temperature in order to estimate the specific attenuation of water vapor [43]. Several techniques can be used to quantify attenuation due to water vapor. Input parameters such as surface water vapor density, $\rho_{s}$, and Integrated Water Vapor Content, $V$, are important and useful for predicting this type of attenuation. Three methodologies for predicting water vapor absorption with different levels of accuracy were suggested by ITU-R P.676-10. An improved ITU-R P.676-10 method of determining water vapor attenuation was suggested in 2017 [44]. This new method enabled researchers to select the prediction model to be used in the absence of local atmospheric profiles. It is documented in Annex 2 of ITU-R P.676-11 [45].

\section{Propagation Impairment Mitigation Techniques (PIMTs)}

It is possible for attenuation to occur on the propagation path in systems working at frequencies beyond $10 \mathrm{GHz}$, as discussed above. The fade margin in such situations may be high. Therefore, propagation impairment mitigation techniques (PIMTs) are crucial for counteracting signal fading and maintaining system reliability. In satellite communication systems, the percentage of time systems offering operational service in a given area is referred to as signal availability. System performance degradation is typically caused by the bit error rate and normalised signal-tonoise ratio $\left(\mathrm{E}_{\mathrm{b}} / \mathrm{N}_{\mathrm{o}}\right)$. A decrease in signal power and increase in noise power will lead to degradation of the signal-tonoise ratio. Signal power decreases when transmitted energy is incompletely received by the receiver because the signal was scattered or absorbed along the transmission path. Therefore, a lower fade marg in is essential in equatorial regions characterised by heavy rain [4], [46].

In general, PIMTs can be categorised into two different classes: (1) those that consider spatial and temporal decorrelation of atmospheric attenuation caused by meteorological phenomena and (2) those that dynamically select the type of transmission modulation/coding. Key techniques include those involving up-linking of power control, adaptive transmission, on-board reconfigurable antenna patterns or spot beam shaping and diversity protection schemes. Diversity protection schemes involve diversity in terms of site, time, orbit and frequency. From the perspective of a satellite operator, diversity protection schemes are the most effective PIMT. However, their selection depends on the propagation scenario at hand. Table 2 summarises the limitations of each PIMT. 
Table 2 - Types of PIMTs and its limiting factor

\begin{tabular}{|c|c|}
\hline PIMT & Limiting Factor \\
\hline Uplink Power Control & Earth station power range \\
\hline Downlink Power Control & $\begin{array}{l}\text { Satellite power range, no offset for } \\
\text { down link fades }\end{array}$ \\
\hline Spot Beam Shaping & Immature research \\
\hline Hierarchical Coding / Hierarchical Modulation & Simultaneous fading in many factors \\
\hline Data Rate Reduction & Rate reduction intolerant applications \\
\hline Site Diversity & Cost \\
\hline Orbital Diversity & Switching between satellites \\
\hline Frequency Diversity & Cost \\
\hline Time Diversity & $\begin{array}{c}\text { Not suitable for real time } \\
\text { applications }\end{array}$ \\
\hline Reconfigurable Antenna Pattern & Cost, complexity \\
\hline
\end{tabular}

In summary, different PIMTs can be selected depending the area in which they are to be applied. PIMTs can also be combined, which may be the best way to maintain QoS.

\section{Conclusion}

This paper briefly reviews atmospheric effects on high-frequency satellite communication as well as the fundamental principles related to these effects and recent important work on scintillation attenuation, rain attenuation, cloud attenuation, water vapor absorption and PIMTs. The review focuses mainly on atmospheric impairments in heavy rain regions, particularly rain and clouds, as these have the biggest impact on radio link fading with respect to the satellite communication and terrestrial links. Future high-throughput satellite communication and 5G mobile systems intended to operate and provide good QoS in heavy rain regions should be able to handle those atmospheric effects in both planning and daily operations.

\section{Acknowledgement}

Authors would like to express deeply gratitude to UTHM funded under “Tier 1" Vot No. H262 and H160.

\section{References}

[1] Ippolito, L. J. (2017). Satellite communications systems engineering. Wiley.

[2] Luini, L., Riva, C. G., Emiliani, L., \& Nessel, J. (2019). Modeling the impact of rain and clouds on earth-space site diversity systems. IEEE Transactions on Antennas and Propagation, 67(1), 475-483.

[3] Zemba, M., Nessel, J., Luini, L., \& Riva, C. (2018). Three years of atmospheric characterization at Ka/Q-band with the NASA/POLIMI Alphasat receiver in Milan, Italy. The 12th European Conference on Antennas and Propagation (EuCAP), 1-5.

[4] Kanatas, A. G., \& Panagopoulos, A. D. (2016). Radio wave propagation and channel modeling for earth-space systems. CRC Press.

[5] Jong, S. L., Riva, C., D'A mico, M., Lam, H. Y., Yunus, M. M., \& Din, J. (2018). Performance of synthetic storm technique in estimating fade dynamics in equatorial Malaysia. International Journal of Satellite Communications and Networking, 36(5), 416-426.

[6] Cuervo, F., Schönhuber, M., Capsoni, C., Lam, H. Y., Jong, S. L., Din, J. B., \& Martellucci, A. (2016). Ka-band propagation campaign in Malaysia-first months of operation and site diversity analysis. The 10th European Conference on Antennas and Propagation (EuCAP), 1-5.

[7] Matricciani, E., Mauri, M., \& Riva, C. (1996). Relationship between scintillation and rain attenuation at 19.77 GHz. Radio Science, 31(2), 273-279.

[8] El-Shami, I. F., Lam, H. Y., Din, J., Elgayar, A. I., \& Alhilali, M. (2018). Tropospheric scintillation with rain attenuation of Ku band at tropical region. Telkomnika, 16(5), 1982-1987.

[9] Adhikari, A., Bhattacharya, A., \& Maitra, A. (2012). Rain induced scintillations and attenuation of Ku-band satellite signals at a tropical location. IEEE Geoscience and Remote Sensing Letters, 9(4), 700-704.

[10] Mertens, D., \& Vanhoenacker-Janvier, D. (2001). Rain fade dependence model of long-term scintillation amplitude distribution at $12.5 \mathrm{GHz}$. Electronics Letters, 37(10), 657-658.

[11] Matricciani, E., Mauri, M., \& Riva, C. (1997). Scintillation and simultaneous rain attenuation at $12.5 \mathrm{GHz}$ to satellite Olympus. Radio Science, 32(5), 1861-1866.

[12] Rogers, D. V, Olsen, R. L., \& Hodge, D. B. (1978). The aRb relation in the calculation of rain attenuation. IEEE Transactions on Antennas and Propagation, 26(2), 318-329. 
[13] ITU-R P.838-3. (2005). Specific attenuation model for rain for use in prediction methods, 1-8.

[14] Lu, C. S., Zhao, Z. W., Wu, Z. Sen, Lin, L. K., Thiennviboon, P., Zhang, X., \& Lv, Z. F. (2018). A new rain attenuation prediction model for the earth-space links. IEEE Transactions on Antennas and Propagation, 66(10), $5432-5442$.

[15] Tokay, A., Bashor, P. G., Habib, E., \& Kasparis, T. (2008). Raindrop size distribution measurements in tropical cyclones. Monthly Weather Review, 136(5), 1669-1685.

[16] Kumar, L. S., Lee, Y. H., \& Ong, J. T. (2011). Two-parameter gamma drop size distribution models for Singapore. IEEE Transactions on Geoscience and Remote Sensing, 49(9), 3371-3380.

[17] Lam, H. Y., Din, J., Lu ini, L., Panagopoulos, A. D., \& Capsoni, C. (2011). Analysis of raindrop size distribution characteristics in Malaysia for rain attenuation prediction. The 30th URSI General Assembly and Scientific Symposium, URSIGASS 2011, 1-4.

[18] Lam, H. Y., Din, J., \& Jong, S. L. (2015). Statistical and physical descriptions of raindrop size distributions in equatorial Malaysia from disdrometer observations. Advances in Meteorology, 2015.

[19] ITU-R P.618-13. (2017). Propagation data and prediction methods required for the design of earth-space telecommunication systems.

[20] Arbesser-Rastburg, B., Martellucci, A., Ondrej, F., \& Erkki, S. (2002). COST action 255: Radiowave propagation modelling for SatCom services at Ku-band and above. Final Report. 53(9), 74-98.

[21] Moupfouma, F. (2009). Electromagnetic waves attenuation due to rain: A prediction model for terrestrial or L.O.S SHF and EHF radio communication links. Journal of Infrared, Millimeter, and Terahertz Waves, 30(6), 622-632.

[22] Silva Mello, L. A. R., \& Pontes, M. S. (2007). Prediction of rain attenuation in terrestrial links with the full rainfall rate distribution. The Second European Conference on Antennas and Propagation (EuCAP), 1-4.

[23] Luini, L., \& Capsoni, C. (2011). MultiEXCELL: A new rain field model for propagation applications. IEEE Transactions on Antennas and Propagation, 59(11), 4286-4300.

[24] Capsoni, C., Fedi, F., Magidtroni, C., Paraboni, A., \& Pawlina, A. (1987). Data and theory for a new model of the horizontal structure of rain cells for propagation applications. Radio Science, 22(3), 395-404.

[25] Capsoni, C., Luini, L., Paraboni, A., Riva, C., \& Martellucci, A. (2009). A new prediction model of rain attenuation that separately accounts for stratiform and convective rain. IEEE Transactions on Antennas and Propagation, 57(1), 196-204.

[26] Yeo, J. X., Lee, Y. H., \& Ong, J. T. (2014). Rain attenuation prediction model for satellite communications in tropical regions. IEEE Transactions on Antennas and Propagation, 62(11), 5775-5781.

[27] Matricciani, E. (1996). Physical-mathematical model of the dynamics of rain attenuation based on rain rate time series and two layer vertical structure of precipitation. Radio Science, 31(2), 281-295.

[28] Jong, S. L., Riva, C., D’A mico, M., Lam, H. Y., Yunus, M. M., \& Din, J. (2018). Performance of synthetic storm technique in estimating fade dynamics in equatorial Malaysia. International Journal of Satellite Communications and Networking, 36(5), 416-426.

[29] Matricciani, E. (1997). Prediction of fade durations due to rain in satellite commun ication systems. Radio Science, 32(3), 935-941.

[30] Cuervo, F., Lam, H. Y., Din, J. Bin, Rivera Castro, J., Schmidt, M., \& Schönhuber, M. (2017). The Joanneum research SatCom Ka and Q band campaigns in Europe and Malaysia. The 11th European Conference on Antennas and Propagation (EUCAP), 1476-1480.

[31] Mandeep, J. S., Hassan, S. I. S., \& Tanaka, K. (2008). Rainfall effects on Ku-band satellite link design in rainy tropical climate. Journal of Geophysical Research, 113(5), 3-8.

[32] Jong, S. L., Lam, H. Y., D’A mico, M., \& Din, J. (2016). Analysis of precipitation characteristics over southem peninsular Malaysia for satellite propagation application. The URSI Asia-Pacific Radio Science Conference (URSI AP-RASC), 1729-1732.

[33] Lam, H. Y., Luini, L., Din, J., Capsoni, C., \& Panagopoulos, A. D. (2012). Assessment of seasonal asia monsoon rain impact on the earth-space propagation in equatorial Kuala Lumpur. International Symposium on Antennas and Propagation (ISAP), 1461-1464.

[34] Lam, H. Y., Luini, L., Din, J., Capsoni, C., \& Panagopoulos, A. D. (2012). Investigation of rain attenuation in equatorial Kuala Lumpur. IEEE Antennas and Wireless Propagation Letters, 11, 1002-1005.

[35] Dissanayake, A., Allnutt, J., \& Haidara, F. (2001). Cloud attenuation modelling for SHF and EHF applications. International Journal of Satellite Communications, 19(3), 335-345.

[36] ITU-R P.840-7. (2017). Attenuation due to clouds and fog.

[37] Salonen, E., \& Uppala, S. (1991). New pred iction method of cloud attenuation. Electronics Letters, 27(12), 11061108

[38] Luini, L., \& Capsoni, C. (2014). Modeling high-resolution 3-D cloud fields for earth-space communication systems. IEEE Transactions on Antennas and Propagation, 62(10), 5190-5199.

[39] Yuan, F., Lee, Y. H., Meng, Y. S., Yeo, J. X., \& Ong, J. T. (2017). Statistical study of cloud attenuation on Kaband satellite signal in tropical region. IEEE Antennas and Wireless Propagation Letters, 16, 2018-2021. 
[40] Manandhar, S., Yuan, F., Lee, Y. H., \& Meng, Y. S. (2016). Weather radar to detect and differentiate clouds from rain events. Proceedings of USNC-URSI Radio Science Meeting (Joint with AP-S Symposium), USNC-URSI, 103-104.

[41] Lyras, N. K., Kourogiorgas, C. I., \& Panagopoulos, A. D. (2017). Cloud attenuation statistics prediction from Kaband to optical frequencies: integrated liquid water content field synthesizer. IEEE Transactions on Antennas and Propagation, 65(1), 319-328.

[42] Yuan, F., Lee, Y. H., Meng, Y. S., Manandhar, S., \& Ong, J. T. (2018). High resolution ITU-R cloud attenuation model for satellite communications in tropical region. IEEE Transactions on Antennas and Propagation.

[43] Lu ini, L., \& Riva, C. G. (2016). Improving the accuracy in pred icting water-vapor attenuation at millimeter-wave for earth-space applications. IEEE Transactions on Antennas and Propagation, 64(6), 2487-2493.

[44] Luini, L., Riva, C., \& Emiliani, L. (2017). Attenuation induced by water vapor along appropriate prediction method, 65(7), 3806-3808.

[45] ITU-R P.676-11. (2016). Attenuation by atmospheric gases.

[46] Panagopoulos, A. D., Arapoglou, P. D. M., \& Cottis, P. G. (2004). Satellite communications at Ku, Ka, and V bands: propagation impairments and mitigation techniques. IEEE Communications Surveys \& Tutorials, $6(3), 2-14$. 